\title{
An improved screw-free method for electrode implantation and intracranial electroencephalographic recordings in mice
}

\author{
Chiping Wu, Marta Wais, TariQ Zahid, AND Qi Wan \\ Toronto Western Research Institute, Toronto, Ontario, Canada \\ AND \\ LiANG ZHANG \\ Toronto Western Research Institute, Toronto, Ontario, Canada \\ and University of Toronto, Toronto, Ontario, Canada
}

\begin{abstract}
We recently developed a glue-based method for the implantation of intracranial electrodes in mice. Our approach is to secure a preconstructed electrode array using a cyanoacrylate-based glue (similar to Krazy Glue). This method is applicable to both young and aging mice and is suitable for long-term electroencephalographic recordings. In the present experiment, we explored whether the glue-based method is capable of securing individual electrodes in addition to securing the electrode array. C57 black mice aged 25-35 days or 13-19 months were operated on under isoflurane anesthesia. Monopolar or bipolar electrodes were inserted independently in the ipsilateral hippocampal CA3 and entorhinal cortical areas, and they were fixed onto the skull using the glue together with dental acrylic, but without anchoring screws. We found that the implanted electrodes were stable and allowed repeat intracranial recordings and electrical stimulation in freely moving mice.
\end{abstract}

Electroencephalographic (EEG) signals are closely related to behavioral states such as sleep-arousal, sensation, movement, and exploration, and the underlying network activities are thought to be important for information processes (Buzsáki, 2006). Abnormal EEG activities are recognizable in a wide range of disease conditions in addition to the physiological EEG patterns (Niedermeyer \& Lopes da Silva, 2005). Intracranial EEG recordings, which offer the opportunity to examine the activity of discrete brain structures, have been used increasingly in experimental studies particularly in mice because of the availability of genetically manipulated mouse models. Although advanced EEG techniques such as multielectrode probes and telemetry systems have been successfully employed in mice (Buzsáki et al., 2003; Jeantet \& Cho, 2003; Korshunov, 2006; Weiergräber, Henry, Hescheler, Smyth, \& Schneider, 2005), long-term intracranial EEG recordings in mice remain a challenge in experimental practice. A pressing technical issue is the manner of securing intracranial electrodes onto the small mouse head.

Screws and dental acrylic are widely used for electrode implantation. Three to four screws are usually twisted through the skull around the area of implantation, and these screws serve as anchors when intracranial electrodes are cemented onto the skull using dental acrylic. Although this method, when used alone or in combination with glue, works well for adult animals (Riban et al., 2002), it may be more problematic when it is employed in young mice or disease models, because their skulls are not strong enough to hold the anchoring screws. The use of anchoring screws is intracranially invasive, moreover, which can increase the likelihood of surgery-related complications such as bleeding, infection, or traumatic injury. These complications can be a major concern in studying disease models, because these animals are often vulnerable to the intracranial surgery.

To minimize the adverse effects that are potentially associated with the use of anchoring screws, we have developed a screw-free, glue-based method for implantation of intracranial electrodes in mice (Wu, Wais, Sheppy, del Campo, \& Zhang, 2008). Our approach is to construct an electrode array, which consists of a plastic base and three assembled electrodes that are arranged according to the stereotaxic coordinates of hippocampal and neocortical recording sites. During surgery, we drill three small holes through the skull to insert these electrodes, and we then secure the electrode array to the skull using a cyanoacrylate-based glue (similar to Krazy Glue). Because the glue distributes evenly in the space between the skull and the plastic base of the electrode array, it holds the electrode array firmly onto the mouse head. When compared with the conventional method of using anchor-

L.Zhang, liangz@uhnres.utoronto.ca 
ing screws, the glue-based approach reduces the time of surgery and implantation-related complications, and it is applicable to mice over a wide age range (from postnatal day 19 up to 26 months).

Although the glue-based method is suitable for longterm intrahippocampal and cortical EEG recordings (Wu et al., 2008), its utilization in other experimental conditions remains untested. For example, it may be difficult to attach an electrode array to curved skull regions. In addition, those who are studying the EEG activities of deep brain structures may prefer to use individual electrodes rather than a preconstructed electrode array. Furthermore, by using individual electrodes for recording and stimulation, one can use electrophysiological criteria (such as EEG activities and/or evoked field potentials) to determine the location of intracranial electrodes. On the basis of these considerations, in the present experiment we explored whether the glue-based method is capable of securing individually positioned electrodes in curved skull regions. Specifically, we used individual monopolar and bipolar electrodes, positioned these electrodes in ipsilateral CA3 and entorhinal areas, and glued these electrodes onto the curved temporal-lobe skull region. We verified the stability of these implanted electrodes by examining regional EEG activities and stimulation-induced field potentials from freely moving animals.

\section{METHOD}

Twelve C57 black mice (Charles River Laboratory, Quebec, Canada) were used in the present experiment. The animals were kept in a vivarium that was maintained at $22^{\circ} \mathrm{C}-23^{\circ} \mathrm{C}$ and that had a $12: 12-\mathrm{h}$ light:dark cycle. Three to four animals were housed in a cage before and after electrode implantation, and a plastic tube and a few pieces of cotton nesting materials were included in the cage. Food and water were available ad libitum. The present experiment was reviewed and approved by the animal care committee of the University Health Network.

Electrodes were made with polyimide-insulated stainless steel wires (outside diameter, 100 or $75 \mu \mathrm{m}$; resistance, $0.87-1.50 \Omega / \mathrm{cm}$; California Fine Wire Company, Grover Beach, CA). Monopolar electrodes consisted of wire (outside diameter, $100 \mu \mathrm{m}$ ) that was cut to approximately $10 \mathrm{~mm}$ in length, with one end of the cut wire soldered to a 6-mm connecting pin that was made from 21-gauge stainless steel tube (Plastics One, Roanoke, VA). Bipolar electrodes were made by twisting wires (outside diameter, $75 \mu \mathrm{m}$ ) and then cutting them to approximately $10 \mathrm{~mm}$ in length. The tips of the twisted wires were left approximately $100 \mu \mathrm{m}$ apart for local stimulation, and the other ends of the twisted wires were soldered to two connecting pins. To improve the hold on the bipolar electrode (see below), one connecting pin was bent into an L shape (Figure 1A).

Surgical procedures were modified from those previously described (in Wu et al., 2008). Mice $25-35$ days $(n=7)$ or $13-19$ months $(n=5)$ of age were operated on for electrode implantation. The animals were anesthetized with isoflurane, using a tabletop anesthesia machine (Model 109, Highland Medical Equipment, Temecula, CA), and a custom-made mouth adapter was used to keep the isoflurane anesthesia at a level of $2 \%$ during the surgical operation. The anesthetized animal was placed onto a stereotaxic frame (David Kopf Instruments, Tujunga, CA). After skin incision and exposure of the skull, three small holes $(<0.5 \mathrm{~mm})$ were made through the skull using a motorized, micromanipulator-assisted driller (Model FM3545, Foredom Electric, Bethel, CT) and a mini drill bit (Part 115603, Ball Mills Carbide, CircuitMedic, Haverhill,
MA). These holes were sufficient for inserting intracranial electrodes but prevented sticky glue from leaking through and into the brain. Monopolar or bipolar electrodes were inserted into the brain individually using micromanipulators. Two electrodes were placed ipsilaterally in the CA3 (bregma, $-1.2 \mathrm{~mm}$; lateral, $1.7 \mathrm{~mm}$; depth, $1.3 \mathrm{~mm}$ ) and entorhinal (bregma, $-4.8 \mathrm{~mm}$; lateral, $2.8 \mathrm{~mm}$; depth, $2.5 \mathrm{~mm}$ ) areas, and a reference electrode was placed in the contralateral cortex (bregma, $-3.8 \mathrm{~mm}$; lateral, $1.8 \mathrm{~mm}$; depth, $0.5 \mathrm{~mm}$; Franklin \& Paxinos, 1997).

After these electrodes were positioned to the desired sites, the glue (Insta-cure 1, BSI Adhesives, Atascadero, CA) was applied to the skull. The glued area was then pasted with freshly mixed dental acrylic (Jet Tooth Shade, Reference No. 1404; hardening time of 6-9 min; Lang Dental Mfg. Co., Inc., Wheeling, IL). The dental acrylic layer was kept at a thickness of approximately $1.5-2 \mathrm{~mm}$ so that it would effectively encase the intracranial electrodes (Figure 1B). Because the glue fastens in several seconds, it was critical to put the dental acrylic onto the glued area quickly in order to achieve a strong bond between the skull and contacting dental acrylic. After the dental acrylic hardened, the electrodes were disengaged from the micromanipulators, and the animal was then released from the stereotaxic frame and allowed to recover in the home cage. Figure $1 \mathrm{C}$ shows the implanted electrodes in an animal that was photographed 3 months after the surgery. In this animal, two bipolar electrodes were implanted in the ipsilateral CA3 and entorhinal cortical areas. For each bipolar electrode, one connecting pin was bent so that it would be held firmly by the dental acrylic (Figure 1D). When implanted headcaps (including electrodes and dental acrylic) were retrieved from sacrificed animals, their total weight was $0.15-0.2 \mathrm{~g}$.

Electrographic signals were recorded using two extracellular amplifiers with extended headstages (Model 300, A-M Systems Inc., Carlsborg, WA), and the ground inputs of these amplifiers were interconnected. The headstages were placed near the animal-housing cage (approximately $10 \mathrm{~cm}$ ) and were connected to implanted electrodes using soft wires and female pins (Wu et al., 2008). The signals were recorded in the frequency band of $0.05-1000 \mathrm{~Hz}$ and were amplified 1,000 times before digitization (digitization rates of 20 or $60 \mathrm{KHz}$; Digidata 1300, Molecular Devices, Union City, CA). Data acquisition, storage, and analyses were completed using pCLAMP software (Version 9, Molecular Devices). For spectral analyses, original data were treated with a digital band-pass filter (Bessel) of 1-500 Hz. The frequency denoted by the main peak of each spectral plot was considered to be the dominant component of the EEG rhythmic signals. For local stimulation, the bipolar electrode was connected to a Grass stimulator (Model S88). Constant current pulses were delivered through an isolation unit every $30 \mathrm{sec}$ and at near maximal intensities $(0.2 \mathrm{msec}, 150 \mu \mathrm{A})$. A train of high-frequency stimuli $(80 \mathrm{~Hz}$ for $1-2 \mathrm{sec}$, at $150 \mu \mathrm{A}$ ) was used to induce seizure-like discharges.

For morphological assessments of implanted electrodes, the animals were anesthetized with a ketamine mixture $(75 \mathrm{mg} / \mathrm{kg}$ of ketamine and $8.25 \mathrm{mg} / \mathrm{kg}$ of xylazine, intraperitoneal injection) and then underwent a transcardiac infusion with $10 \%$ phosphatebuffered formalin solution before decapitation. Brain sections of $50 \mu \mathrm{m}$ thickness were obtained using vibrotom cuts and were examined under a light microscope.

\section{RESULTS}

All animals recovered fully after the surgery and showed neither neurological deficits nor spontaneous seizure activities. EEG recordings were conducted at least 3 days after the surgery and then repeated three to five times over a period of up to 3 months. During the recordings, the animals stayed in home cages and moved freely without restriction. 
A

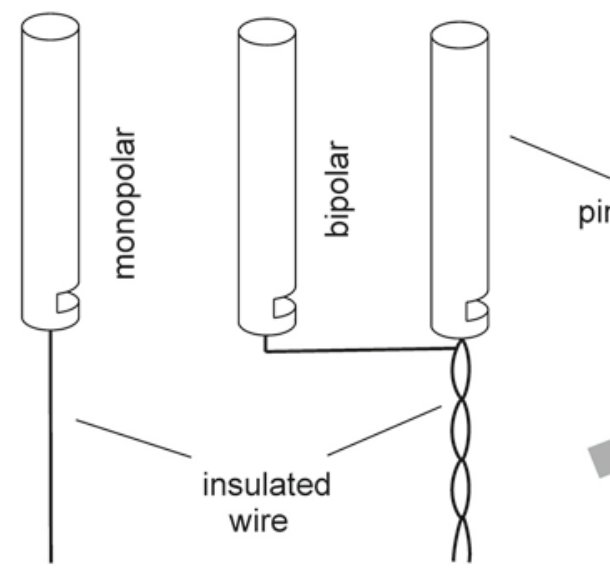

C

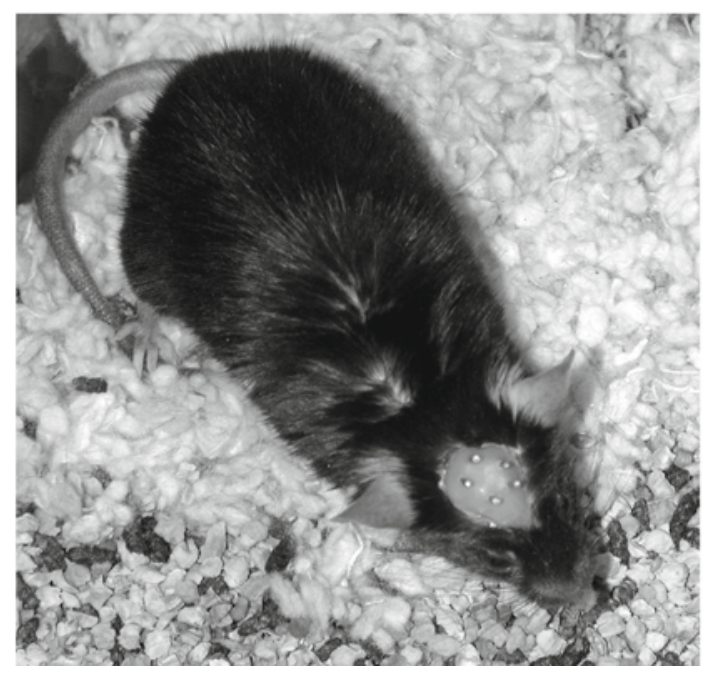

B

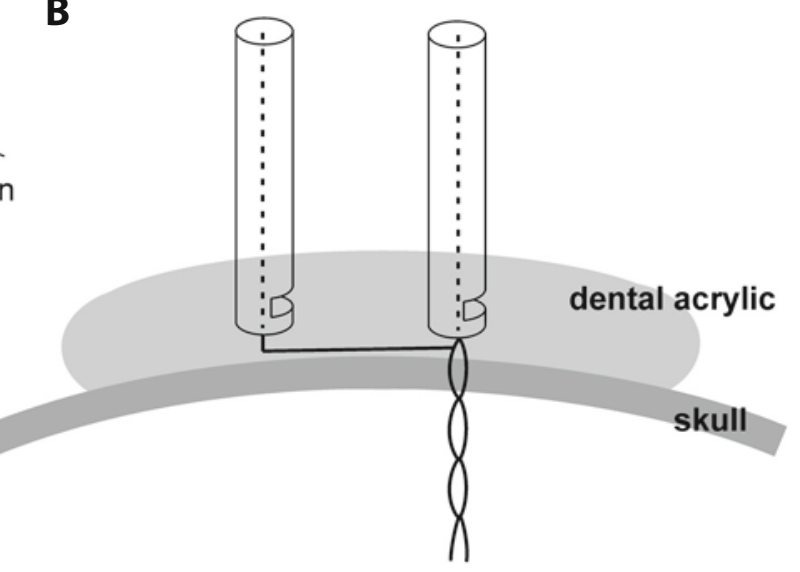

D

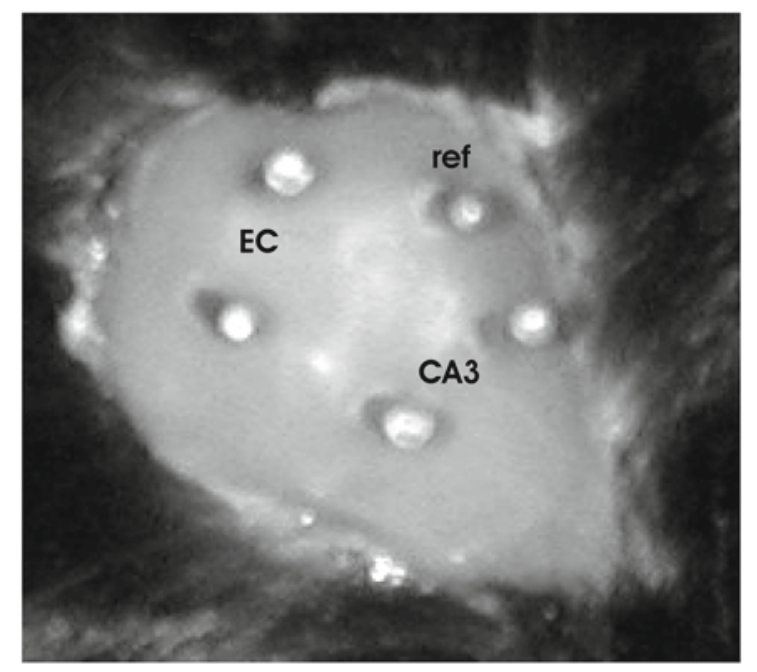

Figure 1. Electrode preparation and implantation. (A) Schematic illustrations of the monopolar and bipolar electrodes. (B) A schematic presentation of the implanted electrodes. (C) An adult mouse 3 months after the implantation. (D) The implantation area, enlarged to show the connecting pins for CA3 and entorhinal (EC) bipolar electrodes and a monopolar reference (ref) electrode.

Physiological EEG activities (Buzsáki, 2006; Buzsáki et al., 2003) were consistently observed in the implanted animals. For example, hippocampal CA3 recordings revealed rhythmic activities at the theta frequency band (theta rhythm, $6.32 \pm 0.31 \mathrm{~Hz} ; n=7$ ) when the animals moved or explored the local environment (Figure 2B); the CA3 EEG was dominated by irregular activities at the delta band $(2.83 \pm 0.41 \mathrm{~Hz} ; n=7)$ while the animals were in awake-immobile or sleep state (Figure 2A). Similar theta rhythms $(7.61 \pm 0.57 \mathrm{~Hz})$ and irregular activities $(2.59 \pm 0.38 \mathrm{~Hz} ; n=9)$ from the entorhinal area were observed, although the entorhinal theta rhythms were not as regular as those observed from the CA3 area (Figures 2A and $2 \mathrm{~B}$ ).

Bipolar electrodes were used for local electrical stimulation of the entorhinal (or CA3) area, and corresponding synaptic field potentials were recorded from the ipsilateral CA3 (or entorhinal) area. In response to the constant stimulation at near-maximal intensity (current pulses of
$0.2 \mathrm{msec}$ and $150 \mu \mathrm{A}$ ), the peak amplitudes of CA3 population spikes and entorhinal field excitatory postsynaptic potentials were $0.28 \pm 0.05 \mathrm{mV}$ and $0.27 \pm 0.04 \mathrm{mV}$ $(n=3)$, respectively. The evoked potentials varied considerably in individual animals, particularly in the CA3 responses, and the amplitudes of consecutively evoked CA3 population spikes (every $30 \mathrm{sec}$ for $10 \mathrm{~min}$ ) ranged from 0.05 to $0.6 \mathrm{mV}$. Although the reasons underlying such variability remain to be determined, these variable responses were associated with a short-term plasticity when evoked by paired stimuli (interstimulation interval of 50 or $250 \mathrm{msec}$ ). As shown in Figures 3A and 3B, when the first evoked responses were small, the second responses were greatly enhanced, whereas the second responses were suppressed if the first evoked responses were relatively large.

Although the singly evoked responses were variable, a train of high-frequency stimuli $(80 \mathrm{~Hz}, 1-2 \mathrm{sec})$ was effective at inducing seizure-like discharges when it was applied to either the CA3 or the entorhinal area (Figures 3A 

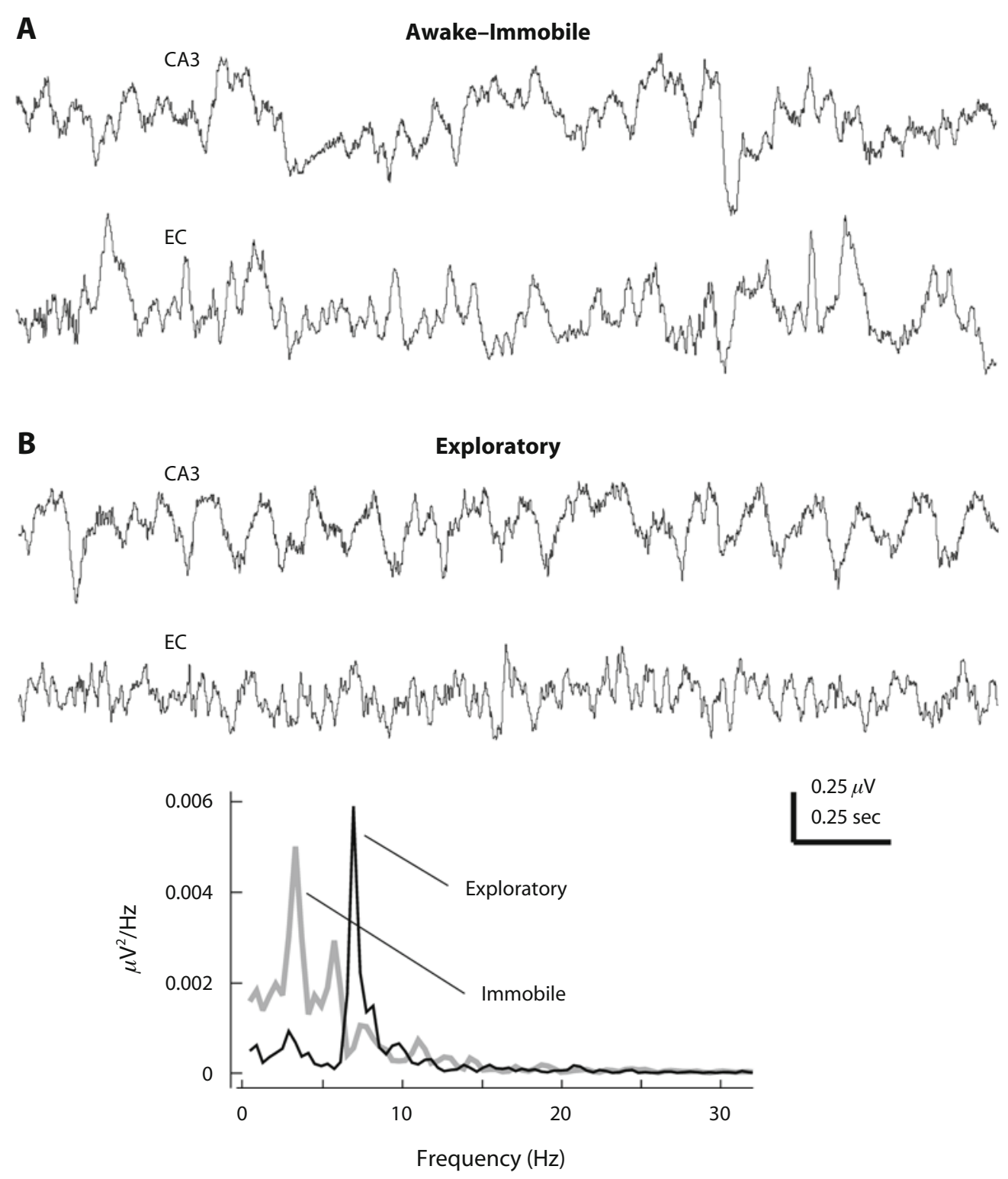

Figure 2. Behaviorally related EEG activities. CA3 and entorhinal (EC) EEG activities were simultaneously recorded from a mouse during awake-immobile (A) and exploratory (B) behaviors, respectively. The animal was implanted at the age of 17 months, and illustrated EEG signals were collected 2 months after the surgery. Corresponding spectral plots were generated from 8-sec data segments, including the traces that are illustrated.

and 3B). These evoked discharges displayed amplitudes up to $2 \mathrm{mV}$ and durations ranging from 7 to $15 \sec (n=3$ mice). In correlation with these electrographic discharges, the animals showed subtle to moderate seizure behaviors such as fast chewing, head nodding, and/or body or forelimb tremors.

To further verify the positions of implanted electrodes, fixed brain sections were obtained from implanted mice, and the traces of implanted electrodes were examined under a light microscope. Of 4 animals that were examined 1-2 months after the surgery, the tracks of implanted electrodes were recognizable in the CA3 and entorhinal areas in 3 mice. One example of the brain sections is pre- sented in Figure 3C, which shows the track of an entorhinal electrode.

\section{DISCUSSION}

We report here that the glue-based method, when used in combination with dental acrylic, is suitable for securing individually positioned electrodes in young and aging mice. Some technical aspects of our method are similar to those that were described in an earlier study (Cain \& Dekergommeaux, 1979), specifically those regarding the use of twisted wire electrodes, connecting pins, and dental acrylic. No anchoring screws were used in the present ex- 

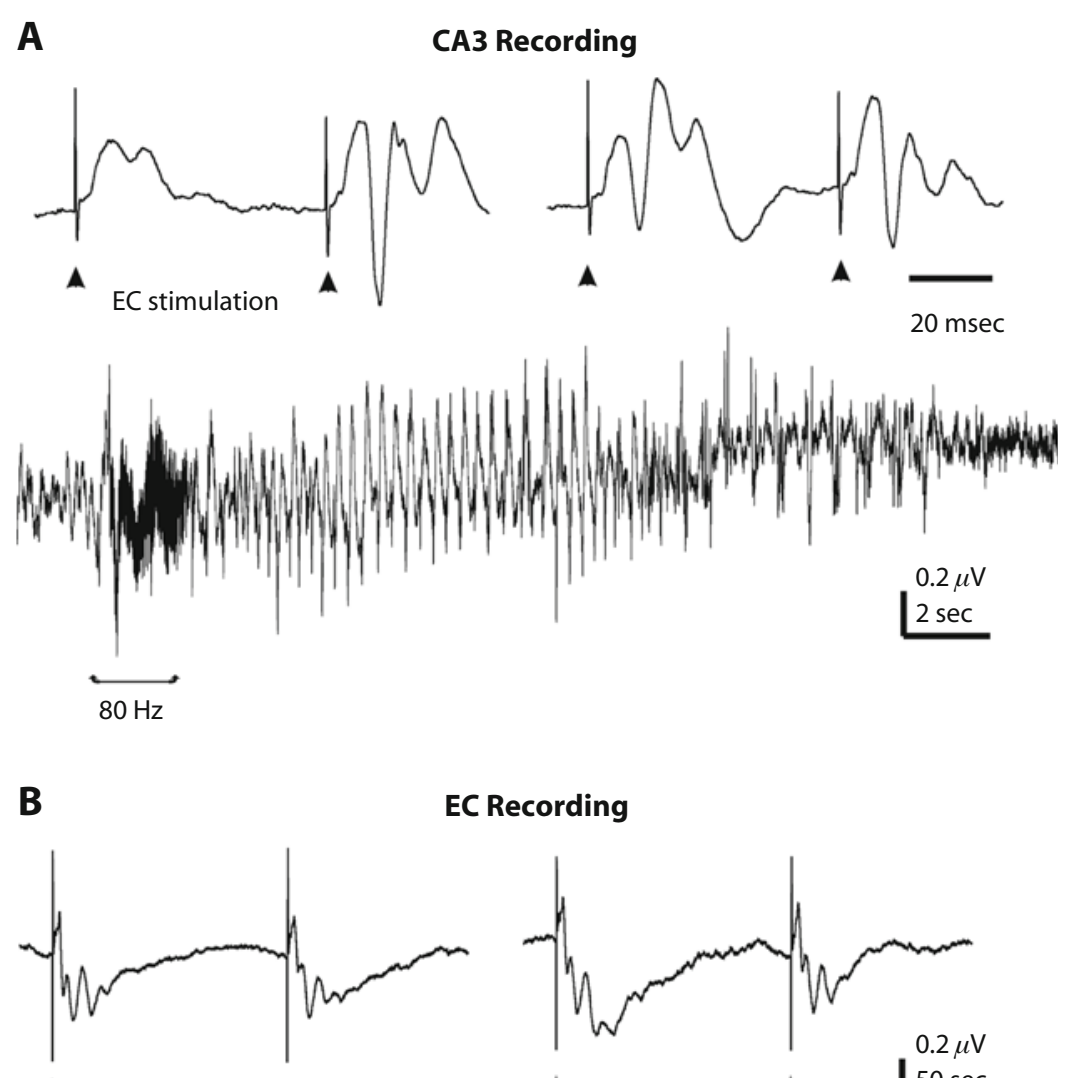

EC Recording
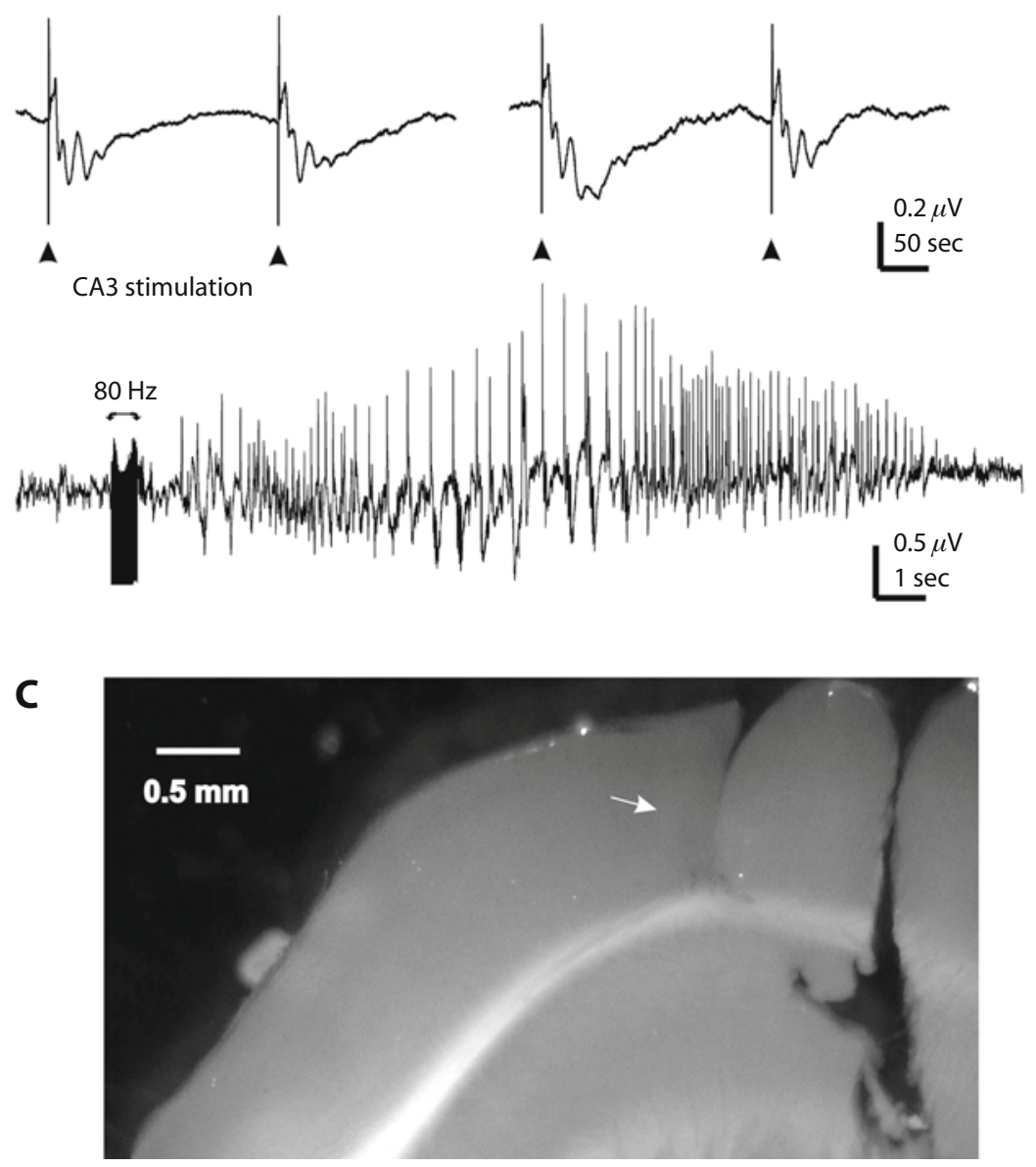

Figure 3. Evoked responses. (A) CA3 responses were evoked by stimulating the entorhinal (EC) area. The animal was implanted at postnatal day 30, and illustrated responses were recorded 7 days after the surgery. The traces showing paired field potentials were averaged from 14 (left) and 6 (right) responses. The seizure-like discharges that were evoked by a train of high-frequency stimuli $(80 \mathrm{~Hz}, 2 \mathrm{sec})$ are shown below. The original data were treated with a band-pass filter (Bessel, 1-500 $\mathrm{Hz}$ ) for illustration purposes. (B) Entorhinal (EC) responses were evoked by stimulating the CA3 area. The animal was implanted at postnatal day 25 and was recorded 3 days later. The traces at the top were averaged from 12 (left) and 8 (right) responses. The seizure-like discharges that were evoked by high-frequency stimuli are shown below. (C) A fixed brain section was collected from a mouse 3 months after the surgery. The track of an implanted entorhinal electrode is denoted by a filled arrow. 
periments, however. Taken together, our present and previous (Wu et al., 2008) results suggest that the glue-based method is convenient and reliable for conducting intracranial recordings and stimulation experiments in mice.

We used isoflurane anesthesia in the present experiment in an attempt to produce both a better control of anesthesia level and a faster postanesthetic recovery process than were produced with the ketamine anesthesia that we used in previous experiments (Wu et al., 2008). It has been shown recently (Tétrault, Chever, Sik, \& Amzica, 2008) that isoflurane anesthesia in cats causes a temporary increase in blood-brain barrier permeability together with alterations in EEG activities. Since our EEG recordings were conducted in the animals that recovered at least 3 days after the surgical operation, the temporary effects of isoflurane anesthesia as reported by Tétrault et al. (2008) were unlikely to have been a major complicating factor in our recording experiments.

Compared with the conventional method using anchoring screws, the glue-based, screw-free approach is less intracranially invasive, because only the small holes $(<0.5 \mathrm{~mm})$ that are necessary for inserting electrodes are drilled through the skull. In addition, since the glue is applied to a relatively large skull area, it provides a strong bond between the skull and the contacting layer of dental acrylic (or the base of the electrode array). Special care should be taken when using the glue in combination with dental acrylic, however. Since the glue fastens in several seconds, whereas dental acrylic hardens in several minutes, it is important to put dental acrylic onto the glued skull area quickly. In addition, a sufficiently thick layer of dental acrylic is needed to encase the intracranial electrodes. Our experience indicates that a dental acrylic layer with a thickness of $1.5-2 \mathrm{~mm}$, when hardened, is appropriate. Because air moisture can make the glue unstable, furthermore, it is preferable to use a glue container only once in order to avoid excessive air exposure.

The use of bipolar electrodes for local electrical stimulation in mice needs further improvement. In the present experiment, the implanted bipolar electrodes were sometimes ineffective at evoking synaptic field potentials, likely as a result of shunting and/or contamination at the tips of the twisted wires. We used relatively thin stainless steel wires (outside diameter, $75 \mu \mathrm{m}$ ) to construct the bipolar electrodes, in an attempt to reduce potential traumatic injury in brain tissues. Because these bipolar electrodes were made from a short piece of twisted thin wires (see Method), however, their strength and stability were potentially inferior to those of electrodes that are made of thicker wires. One possible solution is to somehow bond or glue two thin wires together and have one tip extend beyond the other by about $100 \mu \mathrm{m}$. Further experiments are needed to test this possibility.

\section{AUTHOR NOTE}

Portions of this research were presented at the Sixth International Conference on Methods and Techniques in Behavioral Research. This research was supported by the Natural Science and Engineering Research Council of Canada. Correspondence should be addressed to L. Zhang, Toronto Western Hospital, Room 13-1411, 399 Bathurst Street, Toronto, ON, M5T 258 Canada (e-mail: liangz@uhnres.utoronto.ca).

\section{REFERENCES}

BuzsákI, G. (2006). Rhythms of the brain. Oxford: Oxford University Press.

Buzsáki, G., Buhl, D. L., Harris, K. D., Csicsvari, J., Czéh, B., \& Morozov, A. (2003). Hippocampal network patterns of activity in the mouse. Neuroscience, 116, 201-211.

Cain, D. P., \& Dekergommeaux, S. E. (1979). Electrode implantation in small rodents for kindling and long term brain recording. Physiology \& Behavior, 22, 799-801.

Franklin, K. B. J., \& Paxinos, G. (1997). The mouse brain in stereotaxic coordinates. San Diego: Academic Press.

Jeantet, Y., \& ChO, Y. H. (2003). Design of a twin tetrode microdrive and headstage for hippocampal single unit recordings in behaving mice. Journal of Neuroscience Methods, 129, 129-134.

Korshunov, V. A. (2006). Miniature microdrive-headstage assembly for extracellular recording of neuronal activity with high-impedance electrodes in freely moving mice. Journal of Neuroscience Methods, 158, 179-185.

Niedermeyer, E., \& Lopes da Silva, F. (EdS.) (2005). Electroencephalography: Basic principles, clinical applications, and related fields (5th ed.). Philadelphia: Lippincott Williams \& Wilkins.

Riban, V., Boullleret, V., Pham-Lê, B. T., Fritschy, J.-M., MaresCaUX, C., \& Depaulis, A. (2002). Evolution of hippocampal epileptic activity during the development of hippocampal sclerosis in a mouse model of temporal lobe epilepsy. Neuroscience, 112, 101-111.

Tétrault, S., Chever, O., SiK, A., \& Amzica, F. (2008). Opening of the blood-brain barrier during isoflurane anaesthesia. European Journal of Neuroscience, 28, 1330-1341.

Weiergräber, M., Henry, M., Hescheler, J., Smyth, N., \& SchneiDER, T. (2005). Electrocorticographic and deep intracerebral EEG recording in mice using a telemetry system. Brain Research Protocols, 14, 154-164.

Wu, C., Wais, M., Sheppy, E., del Campo, M., \& Zhang, L. (2008). A glue-based, screw-free method for implantation of intra-cranial electrodes in young mice. Journal of Neuroscience Methods, 171, 126-131.

(Manuscript received October 30, 2008; revision accepted for publication December 16, 2008.) 